\title{
Gastric acid secretion in Chinese with particular reference to the dose of histamine required for maximal stimulation
}

WYE POH FUNG

From the Department of Clinical Medicine, University of Singapore, Singapore 3

SUMMARY Studies of basal and histamine-stimulated gastric acid secretion were performed in 42 controls, in 133 patients with duodenal ulceration, in 57 patients with gastric ulceration, in 13 patients with both duodenal and gastric ulceration, and in 20 patients with gastric carcinoma. All these subjects were Chinese. Statistical analysis of the results showed that all clinical groups differed from the controls in both basal and stimulated secretion. The mean basal acid output of Chinese controls and of patients with duodenal ulcer in most cases did not differ statistically when compared with western series. The basal secretion of Chinese patients with gastric ulceration, however, was statistically higher than in most of the western series. The histamine-stimulated response of Chinese controls and patients with duodenal ulcer was statistically much lower than in all western series with which they were compared. For Chinese patients with gastric ulcer, the stimulated responses were in some instances lower than and in others similar to results obtained in some western series. In contrast to most western reports, the basal and histamine-stimulated secretion in Chinese patients with gastric ulcer was significantly higher than in controls. In the Chinese controls and Chinese patients with peptic ulcers the response after histamine was generally lower than western reports. This was due to the inadequacy of the standard dose of histamine acid phosphate of 0.04 $\mathrm{mg} / \mathrm{kg}$ body weight in Chinese subjects. A dose of $0.06 \mathrm{mg} / \mathrm{kg}$ body weight produced a significantly higher acid output. In the comparatively leaner Chinese subjects, therefore, a dose of histamine acid phosphate of $0.06 \mathrm{mg} / \mathrm{kg}$ is required for maximal stimulation of gastric acid secretion. This agrees well with the results of a similar study in Indian subjects.

Peptic ulceration and gastric carcinoma are common diseases in Chinese patients in Singapore. Gastric acid secretion in controls and in patients with peptic ulceration have been reported from various centres in the western world (Kay, 1953; Lopes, Palmer, and Kirsner, 1959; Sircus, 1960; Baron, 1963a and b; Marks, Banks, Moshal, and Louw, 1963; Grossman, Kirsner, and Gillespie, 1963; Wormsley and Grossman, 1965). These studies represented studies of gastric secretion in western races of Caucasian origin and

Received for publication 20 May 1970. there were few if any reports of gastric secretory studies in Asian races. In India, various studies of gastric acid have been reported (Vakil and Mulekar, 1965; Goyal, Gupta, and Chuttani, 1966; Desai, Borkar, and Jeejeebhoy, 1967; Desai, Antia, Gupte, and Potnis, 1969). The present report concerns gastric secretory studies in Chinese patients living in Singapore, where about $75 \%$ of the $2,000,000$ population are Chinese. In the present investigation the augmented histamine test was used to compare gastric secretion in patients with peptic ulceration and gastric carcinoma with secretion in control subjects. 


\section{Material and Method}

The augmented histamine test as described by Kay (1953) was employed, with some modifications. The tests were done by a specially trained nurse and supervised by the author. Each patient fasted overnight and a double lumen nasogastric tube (size 14 Fr.) was passed in the morning. The nasogastric tube was positioned, without fluoroscopy, by a simple recovery test (Hassan and Hobsley, 1969). Gastric juice was obtained by continuous suction with an electric suction pump and by intermittent hand suction with a syringe. One-hourly basal collections of gastric juice were obtained; collections were also taken after histamine. Thirty minutes before the histamine injection, $50 \mathrm{mg}$ of mepyramine maleate was given intramuscularly. Histamine acid phosphate was given by subcutaneous injection in a dose of $0.04 \mathrm{mg} / \mathrm{kg}$ body weight. The $p \mathrm{H}$ and titratable acidity of each sample was determined by an automatic titrator coupled to a $p \mathrm{H}$ meter (Radiometer, Copenhagen). The $\mathrm{HCl}$ in the gastric juice was titrated against $0.05 \mathrm{~N} \mathrm{NaOH}$ to an

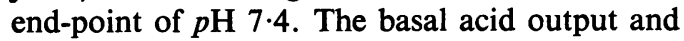
the maximal acid output were thus estimated and expressed as m-equiv $\mathrm{HCl} /$ hour.

Of 265 Chinese patients studied, 42 formed a control group, 133 had duodenal ulceration, 13 had both duodenal and gastric ulceration, 15 had prepyloric ulceration, 42 had corpus gastric ulceration, and 20 had gastric carcinoma. The control group was made up of both normal volunteers (including medical students) and patients with disorders such as skin diseases and epilepsy. These control subjects had no gastrointestinal complaints and most of them had a barium meal examination, which showed no abnormality. In the duodenal ulcer group all the patients had radiological evidence of either a duodenal ulcer crater or chronic duodenal ulcer disease (scarring and deformity of cap). In the group with both duodenal and gastric ulceration, the ulcers were confirmed by barium studies. Of the 57 patients with gastric ulceration, 42 had a corpus ulcer and 15 had prepyloric ulceration. The gastric ulceration in each case was confirmed by barium studies and/or gastroscopy. In the group with gastric malignancy, the diagnosis was confirmed by barium studies, endoscopy, and surgery.

\section{Results}

The age and sex distribution of the patients studied is shown in Table I. The mean basal and maximal acid outputs ( \pm 1 standard deviation) of the controls and the various clinical groups are given in Table II. Statistical analysis of the results showed that all clinical groups differed from the controls $(P<0.05)$ in both basal and stimulated secretion. Duodenal ulcer patients had much higher basal and stimulated acid outputs than controls $(P<0.001)$. Similarly, gastric ulcer patients had higher basal and stimulated acid $\stackrel{?}{?}$ outputs than the control group $(P<0.001)$. Patients with gastric cancer had significantly lower basal and stimulated acid outputs than controls $(P<0.05)$.

In Table III, the results for the Chinese patients studied are compared with the published results $\varnothing$ for western patients previously studied. The ratio is of basal acid output to maximal acid output $\vec{\circ}$ is also shown.

These comparisons show that basal acid $\vec{\omega}$ secretion in the Chinese controls does not differ

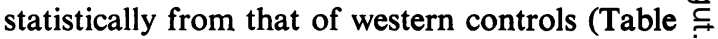
III) except that the basal acid secretion of South $\vec{\exists}$ African patients is higher than all the others (Marks et al, 1963). The basal acid secretion of Chinese patients with duodenal ulcer does not $\mathrm{G}$ differ statistically from results previously pub- 을 lished (Lopes et al, 1959; Sircus, 1960; Grossman, et al, 1963) but is significantly higher $(\mathrm{P}>0.003)$ than results from the series of Baron (1963b) and Wormsley and Grossman (1965). The basal acid secretion of Chinese patients with gastric ulceration is statistically higher $(P>0.003)$ than in all the western groups shown in Table III except for patients from Glasgow (Kay, 1953) and the South African series of Marks et al (1963). The findings in Glasgow and South Africa were similar to those of the present study.

It can also be seen that the histamine-stimulated response of Chinese controls and Chinese patients with duodenal ulcer is significantly $\overrightarrow{\vec{O}}$ lower $(P<0.003)$ than in all the western series (Table III). The histamine-stimulated response of Chinese patients with gastric ulceration is also significantly lower $(\mathrm{P}<0.003)$ than in all western $\stackrel{0}{0}$ series except for the series of Lopes et al (1959), Sircus (1960), and Grossman et al (1963), in which there is no statistical difference with the present study.

The ratio of basal acid output to maximal acid output in each patient was higher in Chinese controls and patients with duodenal and gastric $\frac{D}{O}$ ulcers than in all the western series (Table III).

\section{INCIDENCE OF ANACIDITY}

Anacidity has been variously defined. Failure of $\tilde{\omega}$ gastric juice to become more acid than $p \mathrm{H} 6 \%$ after maximal histamine stimulation (Jones, $\stackrel{O}{\mathcal{O}}$ Gummer, and Lennard-Jones, 1968) was present $\stackrel{\oplus}{\Phi}$ in none of the Chinese controls and patients with? duodenal ulceration but was present in $3.5 \% \frac{0}{\circ}$ of Chinese patients with gastric ulceration and $\stackrel{\vec{D}}{\vec{D}}$

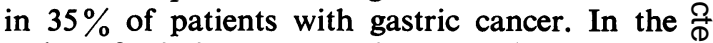
series of Fischermann and Køster (1962), only $\varrho$ one out of 236 patients with benign gastric ulceration had anacidity by this definition (Jones et al, 8 $1968)$ and none at all by their own definition $(p H \geqslant 7)$. In the present series, two of the 57 . patients $(3.5 \%)$ with gastric ulceration and four $\stackrel{F}{\rightleftharpoons}$ 
Gastric acid secretion in Chinese with particular reference to the dose of histamine required

\begin{tabular}{|c|c|c|c|c|c|c|c|c|c|c|}
\hline \multirow[t]{2}{*}{ Group } & \multicolumn{2}{|l|}{ Sex } & \multicolumn{7}{|c|}{ Age Group } & \multirow[t]{2}{*}{ Total } \\
\hline & Male & Female & $<20$ & $20-29$ & $30-39$ & $40-49$ & $50-59$ & $60-69$ & $70+$ & \\
\hline Controls & 21 & 21 & 4 & 20 & 6 & 5 & 3 & 2 & 2 & 42 \\
\hline $\begin{array}{l}\text { Duodenal ulcer } \\
\text { Duodenal and }\end{array}$ & 111 & 22 & 16 & 34 & 29 & 23 & 17 & 11 & 3 & 133 \\
\hline gastric ulcer & 13 & 0 & 1 & 0 & 0 & 4 & 3 & 5 & $\mathbf{0}$ & 13 \\
\hline Prepyloric ulcer & 12 & 3 & 0 & 4 & 2 & 3 & 3 & 2 & 1 & 15 \\
\hline Corpus ulcer & 30 & 12 & 3 & 2 & 3 & 7 & 10 & 11 & 6 & 42 \\
\hline Gastric cancer & 15 & 5 & 0 & 0 & 3 & 1 & 7 & 6 & 3 & 20 \\
\hline Total & 202 & 63 & 24 & 60 & 43 & 43 & 43 & 37 & 15 & 265 \\
\hline
\end{tabular}

Table I Age and sex distribution of patients having augmented histamine tests

\begin{tabular}{|c|c|c|c|c|c|c|c|}
\hline \multirow[t]{2}{*}{ Group } & \multirow[t]{2}{*}{ No. of Patients } & \multicolumn{3}{|c|}{ Basal Acid Output (m-equiv HCl/hr) } & \multicolumn{3}{|c|}{$\begin{array}{l}\text { Histamine-stimulated Acid Output } \\
\text { (m-equiv } \mathrm{HCl} / \mathrm{hr} \text { ) }\end{array}$} \\
\hline & & Mean & $\begin{array}{l}\text { Standard } \\
\text { Deviation }\end{array}$ & $\mathbf{P}$ & Mean & $\begin{array}{l}\text { Standard } \\
\text { Deviation }\end{array}$ & $\mathbf{P}$ \\
\hline \multirow{3}{*}{$\begin{array}{l}\text { Controls } \\
\text { Duodenal ulcer } \\
\text { Duodenal and } \\
\text { gastric ulcer }\end{array}$} & 42 & $1 \cdot 6$ & $1 \cdot 3$ & & $6 \cdot 6$ & $3 \cdot 6$ & \\
\hline & 133 & $5 \cdot 1$ & $3 \cdot 7$ & $<0.001$ & $13 \cdot 3$ & 5.9 & $<0.001$ \\
\hline & 13 & $6 \cdot 4$ & $5 \cdot 1$ & $<0.001$ & $14 \cdot 3$ & $6 \cdot 3$ & $<0.001$ \\
\hline \multirow{2}{*}{$\begin{array}{l}\text { Gastric ulcer } \\
\text { Corpus } \\
\text { Prepyloric } \\
\text { Corpus and } \\
\text { prepyloric }\end{array}$} & $\begin{array}{l}42 \\
15\end{array}$ & $\begin{array}{l}3 \cdot 5 \\
6 \cdot 5\end{array}$ & $\begin{array}{l}3 \cdot 2 \\
5 \cdot 5\end{array}$ & $\begin{array}{l}<0.001 \\
<0.001\end{array}$ & $\begin{array}{r}9 \cdot 8 \\
14.8\end{array}$ & $\begin{array}{l}6 \cdot 8 \\
7.7\end{array}$ & $\begin{array}{r}0.01 \\
<0.001\end{array}$ \\
\hline & 57 & $4 \cdot 3$ & $4 \cdot 1$ & $<0.001$ & $11 \cdot 1$ & $7 \cdot 3$ & $<0.001$ \\
\hline Gastric carcinoma & 20 & 0.8 & $1 \cdot 1$ & $0.01<P<0.05$ & 2.9 & $3 \cdot 4$ & $<0.001$ \\
\hline
\end{tabular}

Table II Gastric acid secretion in Chinese controls and patients with peptic ulceration and gastric carcinoma

\begin{tabular}{|c|c|c|c|c|c|c|c|c|c|c|c|}
\hline \multicolumn{2}{|l|}{ Series } & \multirow[t]{2}{*}{ Method Used } & \multicolumn{3}{|c|}{$\begin{array}{l}\text { Basal Acid Output } \\
\text { (m-equiv } \mathrm{HCl} / \mathrm{hr} \text { ) }\end{array}$} & \multicolumn{3}{|c|}{$\begin{array}{l}\text { Maximal Acid Output } \\
\text { (m-equiv HCl/hr) }\end{array}$} & \multicolumn{3}{|c|}{$\begin{array}{l}\text { Ratio of Basal and Maximal } \\
\text { Acid Output }(\%)^{1}\end{array}$} \\
\hline Place & Author(s) & & Control & $\begin{array}{l}\text { Duodenal } \\
\text { Ulcer }\end{array}$ & $\begin{array}{l}\text { Gastric } \\
\text { Ulcer }\end{array}$ & Control & $\begin{array}{l}\text { Duodenal } \\
\text { Ulcer }\end{array}$ & $\begin{array}{l}\text { Gastric } \\
\text { Ulcer }\end{array}$ & Control & $\begin{array}{l}\text { Duodenal } \\
\text { Ulcer }\end{array}$ & $\begin{array}{l}\text { Gastric } \\
\text { Ulcer }\end{array}$ \\
\hline Glasgow & Kay $(1953)^{2}$ & $\begin{array}{l}\text { Augmented histamine } \\
\text { test }\end{array}$ & $2 \cdot 56$ & $9 \cdot 7$ & $4 \cdot 1$ & $23 \cdot 1$ & $45 \cdot 8$ & $26 \cdot 2$ & $11 \cdot 1$ & $21 \cdot 2$ & $15 \cdot 6$ \\
\hline Edinburgh & Sircus (1960) & $\begin{array}{l}\text { Augmented histamine } \\
\text { test }\end{array}$ & $2 \cdot 5$ & $6 \cdot 0$ & $2 \cdot 1$ & $22 \cdot 4$ & $37 \cdot 6$ & $14 \cdot 6$ & $11 \cdot 1$ & $16 \cdot 0$ & $14 \cdot 4$ \\
\hline UK & $\begin{array}{l}\text { Baron (1963a } \\
\text { and } b)\end{array}$ & $\begin{array}{l}\text { Augmented histamine } \\
\text { test }\end{array}$ & $1 \cdot 3$ & $3 \cdot 6$ & 1.9 & $21 \cdot 6^{3}$ & $42 \cdot 0^{3}$ & $22 \cdot 6^{8}$ & $6 \cdot 0$ & $8 \cdot 6$ & $8 \cdot 4$ \\
\hline S. Africa & $\begin{array}{l}\text { Marks et al } \\
\text { (1963) }\end{array}$ & $\begin{array}{l}\text { Augmented histamine } \\
\text { test }\end{array}$ & $4 \cdot 2$ & $7 \cdot 1$ & $4 \cdot 3$ & $22 \cdot 6$ & $35 \cdot 2$ & $19 \cdot 6$ & $18 \cdot 6$ & $20 \cdot 2$ & $21 \cdot 9$ \\
\hline USA & $\begin{array}{l}\text { Lopes et al } \\
\text { (1959) }\end{array}$ & Histalog test & $2 \cdot 0$ & $5 \cdot 3$ & $1 \cdot 3$ & $12 \cdot 0$ & $20 \cdot 0$ & $14 \cdot 0$ & $16 \cdot 7$ & $26 \cdot 5$ & $9 \cdot 3$ \\
\hline USA & $\begin{array}{l}\text { Grossman et } \\
\text { al (1963) }\end{array}$ & Histalog test & $2 \cdot 4$ & $5 \cdot 3$ & 1.5 & $11 \cdot 6$ & $20 \cdot 0$ & $9 \cdot 6$ & $20 \cdot 7$ & $26 \cdot 5$ & $15 \cdot 6$ \\
\hline USA & $\begin{array}{l}\text { Wormsley and } \\
\text { Grossman (1965) }\end{array}$ & F) & $1 \cdot 8$ & $2 \cdot 1$ & 1.5 & $34 \cdot 4^{3}$ & $42 \cdot 4^{8}$ & $29 \cdot 0^{3}$ & $5 \cdot 2$ & $4 \cdot 9$ & $5 \cdot 1$ \\
\hline India & $\begin{array}{l}\text { Goyal et al } \\
(1966)\end{array}$ & $\begin{array}{l}\text { Augmented histamine } \\
\text { test }\end{array}$ & 2.99 & $5 \cdot 46$ & - & $14 \cdot 48$ & $24 \cdot 38$ & - & $20 \cdot 1$ & $22 \cdot 4$ & - \\
\hline India & $\begin{array}{l}\text { Vakil and } \\
\text { Mulekar (1965) }\end{array}$ & $\begin{array}{l}\text { Augmented histamine } \\
\text { test }\end{array}$ & $2 \cdot 64$ & $5 \cdot 21$ & - & $8 \cdot 74$ & $17 \cdot 85$ & - & $30 \cdot 2$ & $29 \cdot 1$ & - \\
\hline Singapore & Present study & $\begin{array}{l}\text { Augmented histamine } \\
\text { test }\end{array}$ & 1.6 & $5 \cdot 1$ & $4 \cdot 3$ & $6 \cdot 6$ & $13 \cdot 3$ & $11 \cdot 1$ & $27 \cdot 1$ & $40 \cdot 2$ & $39 \cdot 8$ \\
\hline
\end{tabular}

\section{Table III Gastric acid secretion in the present study, in western countries, and in India ${ }^{4}$}

${ }^{1}$ Calculated from mean values except in the present study where the mean ratio was calculated from the value for each patient ${ }^{2}$ Results obtained by converting $\mathrm{mg} \mathrm{HCl} / 45 \mathrm{~min}$ (basal acid output) and $\mathrm{mg} \mathrm{HCl} / 30 \mathrm{~min}$ (maximal acid output) to $\mathrm{m}$-equiv $\mathrm{HCl} / \mathrm{hr}$.

${ }^{3}$ Twice the peak 30-minute output.

‘Mean values only are given. 
of the 20 patients $(20 \%)$ with gastric cancer had a $p \mathrm{H}$ of not less than 7 .

\section{THE PRESENT SERIES}

\section{Dose-response studies for histamine}

Because the histamine-stimulated acid output in Chinese patients was generally so much lower than the output reported in western series, a special study was done to determine whether the dose of histamine acid phosphate $(0.04$ $\mathrm{mg} / \mathrm{kg}$ body weight as recommended by Kay, 1953) in fact constituted a maximal stimulus in Chinese. Secretion stimulated by histamine acid phosphate was measured in 16 patients with the following doses of histamine: $0.04 \mathrm{mg} / \mathrm{kg}, 0.06 \mathrm{mg} / \mathrm{kg}$, and $0.08 \mathrm{mg} / \mathrm{kg}$ body weight. The results are shown in Table IV. When the acid outputs after a dose of $0.04 \mathrm{mg} / \mathrm{kg}$ body weight histamine acid phosphate were compared with outputs after $0.06 \mathrm{mg} / \mathrm{kg}$ body weight, 13 out of the 16 patients showed an increase of acid output with the larger dose of histamine and the mean acid output rose $\Omega$ from 10.7 to $13.8 \mathrm{~m}$-equiv/hour. This rise was statistically significant $(0.001<\mathrm{P}<0.01)$. On com- $\overrightarrow{\vec{c}}$ paring the results after doses of $0.06 \mathrm{mg} / \mathrm{kg}$ body weight with results after doses of $0.08 \mathrm{mg} /$ ? $\mathrm{kg}$, there was no significant difference $(P>0.2) \frac{\text { ज }}{\mathrm{c}}$ When the results of cases 1 to 11 , who all weighed $\overrightarrow{\widetilde{\sigma}}$ less than $60 \mathrm{~kg}$, were analysed, there was again a $\propto$ statistically significant difference between the acid is outputs after $0.04 \mathrm{mg} / \mathrm{kg}$ and $0.06 \mathrm{mg} / \mathrm{kg} \vec{\circ}$ $(0.001<\mathrm{P}<0.01)$ (Table IV). There was again no significant difference between the acid outputs $\vec{\omega}$ after $0.06 \mathrm{mg} / \mathrm{kg}$ and $0.08 \mathrm{mg} / \mathrm{kg}(P>0.2)$.

\section{Influence of sex and weight on acid output}

The mean basal and histamine-stimulated acid outputs ( \pm one standard deviation) in male and $c$ female controls and in patients with duodenal or ulceration and gastric ulceration are shown in $\frac{9}{2}$ Table V. Statistical analysis showed that males secreted more acid than females $(0.01<P<0.05)$

\begin{tabular}{|c|c|c|c|c|c|c|c|}
\hline \multirow[t]{2}{*}{ Case } & \multirow[t]{2}{*}{ Age $(y r)$} & \multirow[t]{2}{*}{$\operatorname{Sex}$} & \multirow[t]{2}{*}{ Weight $(k g)$} & \multirow[t]{2}{*}{ Group } & \multicolumn{3}{|c|}{$\begin{array}{l}\text { Acid Output (m-equiv HCl/hr) of Histamine Acid } \\
\text { Phosphate after Doses (mg/kg body weight) of: }\end{array}$} \\
\hline & & & & & 0.04 & 0.06 & 0.08 \\
\hline $\begin{array}{r}1 \\
2 \\
3 \\
4 \\
5 \\
6 \\
7 \\
8 \\
9 \\
10 \\
11 \\
12 \\
13 \\
14 \\
15 \\
16\end{array}$ & $\begin{array}{l}25 \\
28 \\
37 \\
17 \\
45 \\
31 \\
43 \\
44 \\
18 \\
26 \\
30 \\
61 \\
37 \\
38 \\
30 \\
49\end{array}$ & $\begin{array}{l}\mathbf{M} \\
\mathbf{M} \\
\mathbf{M} \\
\mathbf{M} \\
\mathbf{F} \\
\mathbf{M} \\
\mathbf{M} \\
\mathbf{F} \\
\mathbf{M} \\
\mathbf{M} \\
\mathbf{M} \\
\mathbf{M} \\
\mathbf{M} \\
\mathbf{M} \\
\mathbf{M} \\
\mathbf{M}\end{array}$ & $\begin{array}{l}52 \\
44 \\
53 \\
51 \\
51 \\
50 \\
50 \\
34 \\
59 \\
59 \\
48 \\
60 \cdot 4 \\
90 \\
61 \\
69 \\
64\end{array}$ & $\begin{array}{l}\text { Peptic ulcer } \\
\text { Control } \\
\text { Gastric ulcer } \\
\text { Acute gastritis } \\
\text { Peptic ulcer } \\
\text { Duodenal ulcer } \\
\text { Acute gastritis } \\
\text { Duodenal ulcer } \\
\text { Duodenal ulcer } \\
\text { Peptic ulcer } \\
\text { Duodenal ulcer } \\
\text { Duodenal ulcer } \\
\text { Peptic ulcer } \\
\text { Duodenal ulcer } \\
\text { Peptic ulcer } \\
\text { Peptic ulcer }\end{array}$ & $\begin{array}{r}1 \cdot 6 \\
2 \cdot 7 \\
6 \cdot 5 \\
2 \cdot 5 \\
8 \cdot 3 \\
11 \cdot 3 \\
1 \cdot 2 \\
9 \cdot 0 \\
28 \cdot 1 \\
9 \cdot 5 \\
22 \cdot 0 \\
10 \cdot 1 \\
23 \cdot 2 \\
12 \cdot 5 \\
16 \cdot 1 \\
6 \cdot 5\end{array}$ & $\begin{array}{r}5 \cdot 7 \\
12 \cdot 2 \\
11 \cdot 2 \\
11 \cdot 0 \\
11 \cdot 3 \\
10 \cdot 7 \\
2 \cdot 4 \\
12 \cdot 0 \\
35 \cdot 0 \\
17 \cdot 1 \\
22 \cdot 9 \\
11 \cdot 8 \\
25 \cdot 6 \\
7 \cdot 9 \\
15 \cdot 0 \\
8 \cdot 5\end{array}$ & $\begin{array}{r}2 \cdot 1 \\
12 \cdot 7 \\
8 \cdot 5 \\
11 \cdot 2 \\
- \\
14 \cdot 1 \\
1 \cdot 7 \\
10 \cdot 2 \\
28 \cdot 8 \\
21 \cdot 6 \\
16 \cdot 0 \\
-24 \cdot 4 \\
10 \cdot 2 \\
10 \cdot 0 \\
10 \cdot 9\end{array}$ \\
\hline \multicolumn{8}{|l|}{ Mean } \\
\hline \multicolumn{3}{|c|}{ Cases 1-16 } & & & $\begin{array}{l}10.7 \\
(0.001<P<0.01)\end{array}$ & $\begin{array}{l}13 \cdot 8 \\
(P>0 \cdot 1)\end{array}$ & $\begin{array}{l}13 \cdot 0 \\
(P>0 \cdot 2)\end{array}$ \\
\hline \multicolumn{5}{|c|}{ Cases $1-11$ ( $<60 \mathrm{~kg}$ body weight) } & $\begin{array}{l}9.3 \\
(0.001<P<0.01)\end{array}$ & $\begin{array}{l}13.8 \\
(0.05<P<0.1)\end{array}$ & $\begin{array}{l}12 \cdot 7 \\
(P>0 \cdot 2)\end{array}$ \\
\hline
\end{tabular}

Table IV Gastric acid output after increasing doses of histamine acid phosphate in 16 Chinese subjects of different body weights

\begin{tabular}{|c|c|c|c|c|c|c|c|}
\hline & \multirow[t]{2}{*}{ No. of Patients } & \multicolumn{3}{|c|}{ Basal Acid Output (m-equiv HCl/hr) } & \multicolumn{3}{|c|}{$\begin{array}{l}\text { Histamine-stimulated Acid Output (m-equiv } \\
\mathrm{HCl} / \mathrm{hr} \text { ) }\end{array}$} \\
\hline & & Mean & $S D$ & $\mathbf{P}$ & Mean & $S D$ & $\mathbf{P}$ \\
\hline $\begin{array}{l}\text { Controls } \\
\text { Male } \\
\text { Female }\end{array}$ & $\begin{array}{l}21 \\
21\end{array}$ & $\begin{array}{l}2 \cdot 08 \\
1 \cdot 12\end{array}$ & $\begin{array}{l}1 \cdot 36 \\
1 \cdot 05\end{array}$ & $0.01<P<0.05$ & $\begin{array}{l}8 \cdot 01 \\
5 \cdot 22\end{array}$ & $\begin{array}{l}3 \cdot 55 \\
3 \cdot 07\end{array}$ & $0.001<P<0.01$ \\
\hline $\begin{array}{l}\text { Duodenal ulcer } \\
\text { Male } \\
\text { Female }\end{array}$ & $\begin{array}{r}111 \\
22\end{array}$ & $\begin{array}{l}5 \cdot 35 \\
4 \cdot 12\end{array}$ & $\begin{array}{l}3 \cdot 71 \\
2 \cdot 47\end{array}$ & $\mathbf{P}>0.1$ & $\begin{array}{l}13 \cdot 61 \\
11 \cdot 9\end{array}$ & $\begin{array}{l}5 \cdot 97 \\
4 \cdot 0\end{array}$ & $P>0.2$ \\
\hline $\begin{array}{l}\text { Gastric ulcer } \\
\text { Male } \\
\text { Female }\end{array}$ & $\begin{array}{l}41 \\
16\end{array}$ & $\begin{array}{l}4 \cdot 49 \\
3 \cdot 62\end{array}$ & $\begin{array}{l}4 \cdot 43 \\
3 \cdot 31\end{array}$ & $P>0.4$ & $\begin{array}{c}11 \cdot 7 \\
9 \cdot 74\end{array}$ & $\begin{array}{l}7 \cdot 72 \\
6 \cdot 04\end{array}$ & $P>0.3$ \\
\hline
\end{tabular}

Table V Gastric acid secretion in Chinese males versus females 
in the group of Chinese controls. In Chinese patients with duodenal ulceration or gastric ulceration, however, there was no significant difference in acid output between males and females $(0 \cdot 1<P<0 \cdot 5)$.

Table VI shows the mean basal and histaminestimulated acid outputs in male Chinese with duodenal ulceration and gastric ulceration divided by body weight into three groups. These groups were chosen because most of the patients fell within these weight groupings.

\begin{tabular}{|c|c|c|c|c|c|c|}
\hline \multirow[t]{2}{*}{$\begin{array}{l}\text { Weight } \\
(\mathrm{kg})\end{array}$} & \multicolumn{3}{|c|}{$\begin{array}{l}\text { Basal Acid Output } \\
\text { (m-equiv HCl/hr) }\end{array}$} & \multicolumn{3}{|c|}{$\begin{array}{l}\text { Histamine-stimulated } \\
\text { Acid Output (m-equiv } \\
\text { HCl/hr) }\end{array}$} \\
\hline & Mean & $S D$ & $\mathbf{P}^{1}$ & Mean & $S D$ & $\mathbf{P}$ \\
\hline \multicolumn{7}{|c|}{ Duodenal ulcer } \\
\hline $40-49$ & $6 \cdot 58$ & $4 \cdot 55$ & & $14 \cdot 59$ & 6.02 & \\
\hline $50-59$ & $4 \cdot 10$ & $3 \cdot 77$ & $>0.05$ & 13.59 & 4.99 & $>0.05$ \\
\hline $60-69$ & $4 \cdot 28$ & $2 \cdot 56$ & & 13.91 & 4.83 & \\
\hline \multicolumn{7}{|c|}{ Gastric ulcer } \\
\hline 40-49 & $5 \cdot 37$ & 5.06 & & $11 \cdot 41$ & 4.53 & \\
\hline $50-59$ & 4.82 & $3 \cdot 28$ & $>0.05$ & $19 \cdot 32$ & $8 \cdot 65$ & $>>0.05$ \\
\hline $60-69$ & $7 \cdot 46$ & 5.93 & & $17 \cdot 34$ & 7.90 & \\
\hline
\end{tabular}

Table VI Gastric acid secretion in male Chinese subjects with different body weights

${ }^{2}$ Derived from variance ratio (analysis of variance).

There was no significant difference in acid outputs betsween the various body weights $(P>0.05)$ in male Chinese patients with either duodenal or gastric ulceration.

\section{Influence of age on acid output}

The mean basal and histamine-stimulated acid outputs ( \pm 1 standard deviation) of Chinese patients with duodenal and gastric ulceration in the different age groups are shown in Table VII. In the Chinese patients with duodenal ulcers there was no significant difference in acid output with increasing age, either in basal or histamine-

\begin{tabular}{|c|c|c|c|c|c|c|}
\hline \multirow[t]{2}{*}{ Age (yr) } & \multicolumn{3}{|c|}{$\begin{array}{l}\text { Basal Acid Output } \\
\text { (m-equiv HCl/hr) }\end{array}$} & \multicolumn{3}{|c|}{$\begin{array}{l}\text { Histamine-stimulated } \\
\text { Acid Output (m-equiv } \\
\text { HCl/hr) }\end{array}$} \\
\hline & Mean & $S D$ & $\mathbf{P}^{\mathbf{1}}$ & Mean & $S D$ & $\mathbf{P}$ \\
\hline \multicolumn{7}{|c|}{ Duodenal ulcer } \\
\hline $\begin{array}{l}<20 \\
20-29 \\
30-39 \\
40-49 \\
50-59 \\
60+\end{array}$ & $\begin{array}{l}6 \cdot 59 \\
5 \cdot 81 \\
5 \cdot 12 \\
4.92 \\
3 \cdot 23 \\
4 \cdot 60\end{array}$ & $\begin{array}{l}4 \cdot 16 \\
3 \cdot 76 \\
3 \cdot 29 \\
3 \cdot 42 \\
3.06 \\
2.96\end{array}$ & $>0.05$ & $\begin{array}{l}12 \cdot 84 \\
13 \cdot 54 \\
14 \cdot 12 \\
13 \cdot 34 \\
11 \cdot 16 \\
14 \cdot 34\end{array}$ & $\begin{array}{l}5 \cdot 80 \\
4 \cdot 28 \\
6 \cdot 71 \\
6 \cdot 30 \\
5 \cdot 01 \\
6 \cdot 50\end{array}$ & $=>0.05$ \\
\hline \multicolumn{7}{|c|}{ Gastric ulcer } \\
\hline $\begin{array}{l}<30 \\
30-49 \\
50-59 \\
60+\end{array}$ & $\begin{array}{l}8.97 \\
4.74 \\
3 \cdot 22 \\
2.45\end{array}$ & $\begin{array}{l}5 \cdot 75 \\
3.40 \\
2 \cdot 79 \\
2.91\end{array}$ & $><0.001$ & $\begin{array}{r}16 \cdot 39 \\
11 \cdot 14 \\
11 \cdot 14 \\
8 \cdot 68\end{array}$ & $\begin{array}{l}9 \cdot 24 \\
5 \cdot 63 \\
7 \cdot 44 \\
6 \cdot 42\end{array}$ & \}$>0.05$ \\
\hline
\end{tabular}

Table VII Gastric acid secretion in Chinese subjects (males and females) of different age groups

${ }^{1}$ Derived by analysis of variance. stimulated acid outputs $(\mathrm{P}>0.05)$. However, in Chinese patients with gastric ulcers there was a falling trend with increasing age in both basal and histamine-stimulated acid outputs. When the difference in the levels in the various age groups of Chinese patients with gastric ulcers were tested for significance (by analysis of variance) the differences in basal acid output were highly significant $(\mathrm{P}<0.001)$; those for the histaminestimulated output were not significant $(P>0.05)$.

\section{Discussion}

The findings reported in this study are of importance in three respects; (1) their relationship to similar results in western series; (2) the relative failure of acid secretion to increase adequately with histamine stimulation; and (3) the relationship of these findings to the different patterns of gastric disease seen in Asian and western communities.

\section{COMPARISON OF THE PRESENT RESULTS} WITH THOSE FROM THE WESTERN SERIES The main differences and similarities have already been stated. While it is to be expected that prepyloric ulcers behave like duodenal ulcers in that they have a gastric acid secretion higher than normal, gastric ulcers in general, and corpus ulcers in particular, have been reported to be associated with acid outputs lower than normal (Sircus, 1960; Grossman et al, 1963; Wormsley and Grossman, 1965). In the present study, not only the patients with prepyloric ulcer but also those with corpus ulceration had mean acid outputs significantly higher than control subjects $(\mathrm{P}<0.01)$. The group of 57 patients with gastric ulceration (Table II) thus had mean acid outputs significantly higher than the control group $(P<0.001)$. It would appear therefore that Chinese patients with gastric ulceration have a gastric acid secretion significantly higher than normal, while in most western groups gastric ulceration is associated with an acid secretion lower than normal. There are most probably ethnic reasons for this difference.

RISE IN ACID SECRETION AFTER HISTAMINE AND THE HISTAMINE DOSE REQUIRED FOR MAXIMAL STIMULATION It has been shown that the acid output after histamine correlates with the parietal cell mass (Card and Marks, 1960). Consequently, the results in the present study taken with the failure of acid output to rise to the levels observed in western patients could be accounted for in Chinese patients by: (1) a smaller parietal cell mass of which a greater proportion actively secretes under basal conditions; (2) a normal 
parietal cell mass that is less responsive to histamine-also if relatively refractory to histamine stimulation, it is normally reactive to the stimuli that control basal acid secretion; and (3) a dose $(0.04 \mathrm{mg} / \mathrm{kg}$ body weight $)$ of histamine acid phosphate being given which is insufficient for maximal stimulation.

It has also been reported that the maximal acid output in Indian controls and in subjects with duodenal ulceration was lower than that reported in Western subjects (Vakil and Mulekar, 1965; Goyal et al, 1966).

Desai et al (1967) have shown that in Indian subjects weighing less than $60 \mathrm{~kg}$, a dose of $0.04 \mathrm{mg} / \mathrm{kg}$ body weight of histamine acid phosphate was inadequate for maximal stimulation of gastric acid, since higher doses showed a significant rise in acid output. Desai and his co-workers further showed that in Indians weighing between 40 and $60 \mathrm{~kg}$, doses of histamine acid phosphate of $0.05 \mathrm{mg} / \mathrm{kg}$ for controls and 0.06 $\mathrm{mg} / \mathrm{kg}$ for duodenal ulcer patients were required for maximal stimulation, and that supramaximal doses of histamine acid phosphate resulted in a reduction of acid output in some subjects.

The results of the present study are in agreement with these findings, since it was found that in Chinese patients a dose of histamine acid phosphate of $0.06 \mathrm{mg} / \mathrm{kg}$ body weight is required for maximal stimulation of gastric acid secretion. A dose of histamine acid phosphate of 0.04 $\mathrm{mg} / \mathrm{kg}$, while adequate for most western subjects, would thus be a submaximal dose in Chinese subjects. The majority of Chinese subjects in this study weighed between $40 \mathrm{~kg}$ and $70 \mathrm{~kg}$ (mean $52 \mathrm{~kg}$ ). This compares well with the series of Indians the majority of whom weighed between $40 \mathrm{~kg}$ and $65 \mathrm{~kg}$, with a mean of $53 \mathrm{~kg}$ for males (Desai et al, 1967 and 1969). The poorer response to histamine stimulation in the present study is thus due to the inadequacy of a dose of 0.04 $\mathrm{mg} / \mathrm{kg}$ body weight.

It is therefore recommended that a dose of histamine acid phosphate of $0.06 \mathrm{mg} / \mathrm{kg}$ be given for maximal stimulation of gastric acid secretion in Chinese subjects. As in the Indian series (Desai et al, 1969), it was also found in the present study that supramaximal doses of histamine acid phosphate (above $0.06 \mathrm{mg} / \mathrm{kg}$ ) resulted in a reduction of acid output in some of the Chinese subjects (Table IV). This difference in dose requirement between Indians and Chinese on the one hand and western subjects on the other may be related to the difference in body weight and, more specifically, to the partition of gross weight between fat and lean body mass (Makhlouf, 1969). Unfortunately it is not known to what extent histamine acid phosphate is distributed in the body fat. If only a negligible amount is distributed in the body fat then a dose of 0.04 $\mathrm{mg} / \mathrm{kg}$ in western subjects (average weight $69 \mathrm{~kg}$ ) with a normal amount of body fat would result in an effective concentration of 0.05 to $0.055 \mathrm{mg}$ / $\mathrm{kg}$ lean body mass (Makhlouf, 1969). This is in agreement with the dose proposed by Desai et al (1969) for the leaner Indian subjects (average weight $53 \mathrm{~kg}$ ) and with the dose recommended in the present study for the leaner Chinese subjects (average weight $52 \mathrm{~kg}$ ).

As a dose of histamine acid phosphate of $0.04 \mathrm{mg} / \mathrm{kg}$ is submaximal for Chinese subjects, the histamine-stimulated acid outputs in the present series are thus not true maximal acid outputs. This explains the higher ratios of basal to maximal acid outputs for controls and peptic ulcer groups in the present series compared to the results in western series (Table III.) This also accounts for the relatively high incidence of patients having a basal to maximal acid output ratio above $60 \%$ in the present series $(12 \%$ controls, $21.9 \%$ duodenal ulcer patients, $25.4 \%$ gastric ulcer patients).

\section{RELATIONSHIP OF THE SECRETORY}

PATTERN OBSERVED AND THE PATTERN OF GASTRIC DISEASE SEEN IN CHINESE

\section{PATIENTS}

Chinese and Japanese are prone to gastric ulceration and gastric carcinoma. In Japan, gastric cancer is the most common tumour in men, the incidence being approximately four times that found in the United States (Morrissey, Tanaka, and Thorsen, 1967). Gastric ulcer was the cause of death in 32.1 in every 100,000 in Japan as compared to 6.7 per 100,000 in England and 4.1 per 100,000 in the United States (Segi, Fujisaku, and Kurihara, 1958). In Singapore, where about $75 \%$ of the population are Chinese, gastric carcinoma is the third commonest cancer for both sexes and the second commonest cancer for males (Annual Report, Singapore Cancer Registry, 1950-1964). Out of 223 Chinese patients in Singapore with proven peptic ulceration $31.4 \%$ had gastric ulceration. Gastric ulceration is characterized by normal or slightly reduced acid secretion in western communities. In this study, the Chinese patient with gastric ulcer had a high basal and stimulated acid secretion and consequently it may be assumed that in this group the role of acid and pepsin is relatively more important than decreased mucosal resistance in the aetiology of peptic ulcer. The cancer group in this study had a low acid secretion similar to that observed in the western series (Grossman et al, 1963). The control group did not have a low acid secretory rate despite the ethnic predisposition to gastric cancer.

The author is grateful to Professor D. W. Piper and Dr A. R. Cooke for valuable advice in the preparation of this report, to F. C. Chong for laboratory assistance, to the China Medical Board for donating the automatic titrator and the 
$p \mathrm{H}$ meter, and to $\mathrm{Mr} \mathrm{C}$. Y. Tye and Dr Leonard Tan for assistance with the statistics.

\section{References}

Annual Report (1950-1964). Unpublished data from the Cancer Registry Singapore Institute of Pathology.

Baron, J. H. (1963a). An assessment of the augmented histamine test in the diagnosis of peptic ulcer. Gut, 4, 243-253.

Baron, J. H. (1963b) Studies of basal and peak acid output with an augmented histamine test. Gut, 4, 136-144.

Card, W. I., and Marks, I. N. (1960). The relationship between the acid output of the stomach following 'maximal' histamine stimulation and the parietal cell mass. Clin. Sci., 19, 147-163.

Desai, H. G., Antia, F. P., Gupte, U. V., and Potnis, P. R. (1969). Dose of histamine for maximal stimulation of gastric acid secretion. Modified subcutaneous histamine test. Gastroenterology, 57, 636-640.

Desai, H. G., Borkar, A. V., and Jeejeebhoy, K. N. (1967). Dose-weight relationship of histamine for maximal stimulation of gastric acid secretion. Gastroenterology, 53, 712-718.

Fischermann, K., and Køster, K. H. (1962). The augmented histamine test in the differential diagnosis between ulcer and cancer of the stomach. Gut, 3, 211-218.

Goyal, R. K., Gupta, P. S., and Chuttani, H. K. (1966). Gastric acid secretion in Indians with particular reference to the ratio of basal to maximal acid output. Gut, 7, 619-623.

Grossman, M. I., Kirsner, J. B., and Gillespie, I. E. (1963). Basal and histalog-stimulated gastric secretion in control subjects and in patients with peptic ulcer or gastric cancer. Gastrienterology, 45, 14-26.
Hassan, M. A., and Hobsley, M. (1969). Positioning of nasogastric tubes without fluoroscopy. Gut, 9, 728 .

Jones, F. A., Gummer, J. W. P., and Lennard-Jones, J. E. (1968). Clinical Gastroenterology, p. 841. Blackwell, Oxford.

Kay, A. W. (1953). Effect of large doses of histamine on gastric secretion of $\mathrm{HCl}-$ an augmented histamine test. Brit. med. J., 2, 77-80.

Lopes, R. W., Palmer, W. I., and Kirsner, J. B. (1959). Pyloric ulcer with specific reference to gastric secretory pattern. Gastroenterology, 36, 790-795.

Makhlouf, G. (1969). The highest effective dose of histamine for gastric secretion. Gastroenterology, 57, 766.

Marks, I. N., Bank, S., Moshal, M. G., and Louw, J. H. (1963). The augmented histamine test. A review of 615 cases of gastroduodenal disease. S. Afr. J. Surg., 1, 53-59.

Morrisey, J. F., Tanaka, Y., and Thorsen, W. B. (1967). Gastroscopy. A review of the English and Japanese literature. Gastroenterology, 53, 456-476.

Segi, M., Fujisaku, S., and Kurihara, M. (1958). Mortality for gastric and duodenal ulcer in countries and its geographical correlation to mortality for gastric and intestinal cancer. Schweiz. Z. allg. Path., 22, 777-784. (Quoted by G. Watkinson (1961). In Modern Trends in Gastroenterology, 3rd series, edited by W. I. Card, pp. 24-25. Butterworths, London.)

Sircus, W. (1960). The aetiology of peptic ulcer. In Peptic Ulcer: a Symposium for Surgeons, edited by C. Wells and J. Kyle, ch. 2, pp. 11-36. Livingstone, Edinburgh and London.

Vakil, B. J., and Mulekar, A. M. (1965). Studies with the maximal histamine test. Gut, 6, 364-371.

Wormsley, K. G., and Grossman, M. I. (1965). Maximal histalog test in control subjects and patients with peptic ulcer. Gut, 6, 427-435. 